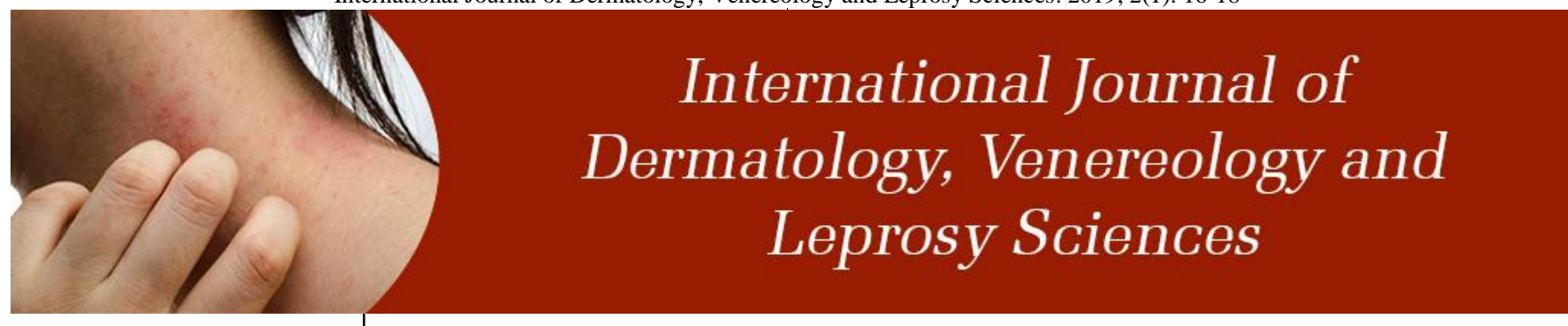

E-ISSN: 2664-942X

P-ISSN: 2664-9411

www.dermatologypaper.com/

Derma 2019; 2(1): 16-18

Received: 15-11-2018

Accepted: 18-12-2018

Shiksha Pandit

Department of Dermatology,

Department Of Community

Medicine, BMCRI, Bengaluru,

Karnataka, India
Corresponding Author:

Shiksha Pandit

Department of Dermatology,

Department Of Community

Medicine, BMCRI, Bengaluru,

Karnataka, India

\section{Assessment of antimelanocyte antibodies in patients of vitiligo}

\section{Shiksha Pandit}

DOI: https://doi.org/10.33545/26649411.2019.v2.i1a.20

\begin{abstract}
Introduction: Vitiligo is a non contagious acquired pigmentation disorder characterized by sharply defined white patches of variable shape and dimensions, increasing in size and number with time. The present study assessed anti-melanocyte antibodies (AMAs) in patients of vitiligo.

Materials \& Methods: The present study was conducted on 72 cases of vitiligo of both genders. The severity of vitiligo was assessed according to the Vitiligo Area Scoring Index (VASI). AMA was measured by human melanocyte antibody enzyme-linked immunosorbent assay (ELISA) Kit.

Results: In group I (cases), there were 40 males and 32 females and in group II (control), there were 34 males and 38 females. The mean VASI score in males was 8.13 and in females was 8.45 , duration of disease was 6.24 years in males and 6.17 years in females. The difference was significant $(P<0.05)$. The mean AMA in group I was $14.7 \mathrm{mg} / \mathrm{ml}$ and in group II was $5.2 \mathrm{mg} / \mathrm{ml}$. The difference was significant $(P<0.05)$.

Conclusion: Authors found that there was significantly higher level of antimelanocyte antibodies (AMA) in patients than control.
\end{abstract}

Keywords: Antimelanocyte, Antibodies, Vitiligo

\section{Introduction}

Vitiligo is a non contagious acquired pigmentation disorder characterized by sharply defined white patches of variable shape and dimensions, increasing in size and number with time. The histological picture shows loss of melanocytes and melanin in the white patches and an inconstant lympho-mononuclear infiltrate in the advancing margins of vitiligo ${ }^{[1]}$.

The clinical picture consists of one or more well demarcated and white maculae, progressing in size and number. They are asymptomatic generally. The lesions usually appear on sun exposed or constitutionally hyperpigmented areas or on sites of stretch and pressure (face, dorsum of hands and fingers, external genitalia, knees and elbows). The margins of the patches are often hyperpigmented; hypopigmented areas sometimes occur together with the depigmented lesions and the normally pigmented skin (trichrome vitiligo). Rarely an inflammatory border may be found around the vitiligo patch resulting in a raised and erythematous edge (inflammatory vitiligo). Poliosis circumscripta, as well as canities and premature graying, can be observed; mucosae are rarely involved ${ }^{22]}$.

The development of autoimmune-associated vitiligo could be attributed to either a $\mathrm{T}$ cellbased pathomechanism in cases of localized disease or natural killer cells, $\mathrm{T}$ cells, and antibodies in cases of diffuse vitiligo. Most vitiligo susceptibility loci identified through genome-wide association studies encode immunomodulatory proteins, supporting the autoimmune pathogenesis of vitiligo ${ }^{[3]}$.

Patients with vitiligo have an increased concentration of circulating autoantibodies that are specific to melanocyte cytoplasm and surface antigens compared with normal individuals that related to the activity of the disease. On the other hand, it was reported that melanocyte antigen-specific antibodies cannot be used as markers for recent disease activity in patients with vitiligo ${ }^{[4]}$. The present study assessed antimelanocyte antibodies (AMAs) in patients of vitiligo.

\section{Materials \& Methods}

The present study was conducted in the department of Dermatology. It comprised of 72 cases 
of vitiligo of both genders. Equal number of healthy subjects was also included. All were informed regarding the study and written consent was obtained. Ethical clearance was taken before starting the study.

General information such as name, age, etc. was noted. The severity of vitiligo was assessed according to the Vitiligo Area Scoring Index (VASI). AMA was measured by human melanocyte antibody enzyme-linked immunosorbent assay (ELISA) Kit. It detects Melanocyte protein 17 (premelanosome protein 17). These antibodies are specific for a melanocyte-specific type 1 transmembrane glycoprotein. Results thus obtained were subjected to statistical analysis using chi- square test. $\mathrm{P}$ value less than 0.05 was considered significant.

\section{Results}

Table I: Distribution of subjects

\begin{tabular}{|c|c|c|}
\hline Gender & Group I (Cases) & Group II (Control) \\
\hline Male & 40 & 34 \\
\hline Female & 32 & 38 \\
\hline
\end{tabular}

Table I shows that in group I (cases), there were 40 males and 32 females and in group II (control), there were 34 males and 38 females.

Table II: Assessment of parameters

\begin{tabular}{|c|c|c|c|}
\hline Parameters & Male & Female & P value \\
\hline VASI (Mean) & 8.13 & 8.45 & 0.83 \\
\hline Duration of disease (Years) & 6.24 & 6.17 & 0.92 \\
\hline
\end{tabular}

Table II, graph I shows that mean VASI score in males was 8.13 and in females was 8.45 , duration of disease was 6.24 years in males and 6.17 years in females. The difference was significant $(P<0.05)$.

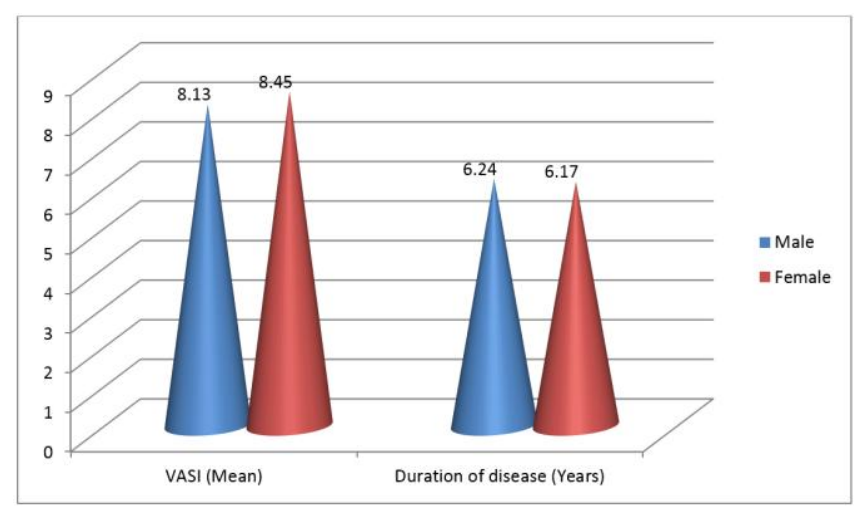

Graph I: Assessment of parameters

Table III: Assessment of AMA in both groups

\begin{tabular}{|c|c|c|}
\hline AMA & Mean & P value \\
\hline Group I & 14.7 & \multirow{2}{*}{0.01} \\
\hline Group II & 5.2 & \\
\hline
\end{tabular}

Table III shows that mean AMA in group I was $14.7 \mathrm{mg} / \mathrm{ml}$ and in group II was $5.2 \mathrm{mg} / \mathrm{ml}$. The difference was significant $(P<0.05)$.

\section{Discussion}

Vitiligo is an acquired skin disorder characterized by white and depigmented patches enlarging and becoming more numerous with time. It is due to a disappearance of functioning melanocytes and loss of melanin in the epidermis. The condition can be cosmetically disfiguring and the lesional skin is thus more sensitive to sunburns. It affects $0.1-2 \%$ of the world's population, irrespective of gender and race ${ }^{[5]}$. Etiology is still unknown, but some evidence based pathogenetic hypotheses have been proposed to explain the loss of melanocytes in epidermis. A positive family history for vitiligo is reported. Actually, family clustering of cases is not uncommon, since about $20 \%$ of patients have at least one affected first-degree relative, with a non-Mendelian pattern suggestive of multifactorial, polygenic inheritance; segregation analyses suggest the involvement of multiple interacting genes in different populations. Several genes and chromosomal regions have been implicated in susceptibility to vitiligo, but none has been confirmed so far. In addition, several HLA abnormalities have been associated with vitiligo, including association with Dr4, B13, BW35, and A30 ${ }^{[6]}$. The present study assessed antimelanocyte antibodies (AMAs) in patients of vitiligo.

In present study, in group I (cases), there were 40 males and 32 females and in group II (control), there were 34 males and 38 females. The mean VASI score in males was 8.13 and in females was 8.45 , duration of disease was 6.24 years in males and 6.17 years in females.

Autoimmune hypothesis proposes that an immune system disorder results in destruction of melanocytes. It is first supported by the frequent observation that several autoimmune disorders (thyroid diseases, Sutton nevi, juvenile diabetes mellitus, pernicious anemia and Addison's disease) are associated with vitiligo ${ }^{[7]}$. A significant association of vitiligo was demonstrated with thyroid dysfuntion and/or thyroid antibodies in particular. Concerning humoral immunity, antibodies to surface and cytoplasmic antigens of melanocytes have been found in vitiligo patients, mainly belonging to the IgG class. The autoantigens most frequently identified are antigens related to HLA class I molecules, tyrosinase, tyrosinase-related protein (TRP)-1 and TRP-2 (the last three are melanocyte specific antigens) ${ }^{[8]}$.

El-Gayyar et al. ${ }^{[9]}$ in their study severity of vitiligo was assessed according to the Vitiligo Area Scoring Index (VASI). AMA, C3 and C4, and ANA serum levels were measured for patients and controls. It was found that ANA, AMA, and C4 levels were significantly higher in the sera of patients than in controls. ANA, AMA, and C4 serum levels showed significant positive correlations with VASI score.

We observed that mean AMA in group I was $14.7 \mathrm{mg} / \mathrm{ml}$ and in group II was $5.2 \mathrm{mg} / \mathrm{ml}$. There was significantly higher level of AMA in patients than control. Both the incidence and serum concentrations of these antibodies increases with the extent of the disease being detected in $50 \%$ of patients with minimal vitiligo. Li et al. ${ }^{[10]}$ proved that hydroxychloroquine protects melanocytes from autoantibody-induced injury by reducing the binding of antigen-antibody complexes reversing the activities of ADCC and CDC in vitro. On the other hand, Kroon et al. ${ }^{[11]}$ found no correlation between the presence of antibodies and recent disease activity or other clinical characteristics such as age, gender, extension, and duration of vitiligo.

\section{Conclusion}

Authors found that there was significantly higher level of 
antimelanocyte antibodies (AMA) in patients than control.

\section{References}

1. Alikhan A, Felsten LM, Daly M, Petronic-Rosic V. Vitiligo: A comprehensive overview part I. Introduction, epidemiology, quality of life, diagnosis, differential diagnosis, associations, histopathology, etiology, and work-up. J Am Acad Dermatol. 2011; 65:473-91.

2. Rodrigues M, Ezzedine K, Hamzavi I, Pandya AG, Harris JE; Vitiligo Working Group. New discoveries in the pathogenesis and classification of vitiligo. J Am Acad Dermatol. 2017; 77:1-3. 3.

3. Boniface K, Seneschal J, Picardo M, Taïeb A. Vitiligo: Focus on clinical aspects, immunopathogenesis, and therapy. Clin Rev Allergy Immunol. 2018; 54:52-67.

4. Choi D, Isedeh P, Hamzavi IH. Vitiligo: A review of the pathogenesis. J Egypt Womens Dermatol Soc. 2014; 11:145-8.

5. Jin Y, Andersen G, Yorgov D, Ferrara TM, Ben S, Brownson KM et al. Genome-wide association studies of autoimmune vitiligo identify 23 new risk loci and highlight key pathways and regulatory variants. Nat Genet 2016; 48:1418-24.

6. Lin X, Tian H, Xianmin M. Possible roles of B lymphocyte activating factor of the tumour necrosis factor family in vitiligo autoimmunity. Med Hypotheses 2011; 76:339-42.

7. Nordlund J, Lerner AB. Vitiligo. It is important. Arch Dermatol 118:5-8, 1982.

8. Koga M, Tango T. Clinical features and course of type A and type B vitiligo. Brit J Dermatol. 1988; 118:223228.

9. El-Gayyar MA, Helmy ME, Amer ER, Elsaied MA, Gaballah MA. Antimelanocyte antibodies: A possible role in patients with vitiligo. Indian J Dermatol. 2020; 65:33-7.

10. Li DG, Hu WZ, Ma HJ, Liu W, Yang QQ, Zhao G. Hydroxychloroquine protects melanocytes from autoantibody-induced injury by reducing the binding of antigen-antibody complexes. Mol Med Rep. 2016; 14:1275-82.

11. Kroon MW, Kemp EH, Wind BS, Krebbers G, Bos JD, Gawkrodger DJ et al. Melanocyte antigen-specific antibodies cannot be used as markers for recent disease activity in patients with vitiligo. J Eur Acad Dermatol Venereol. 2013; 27:1172-5. 